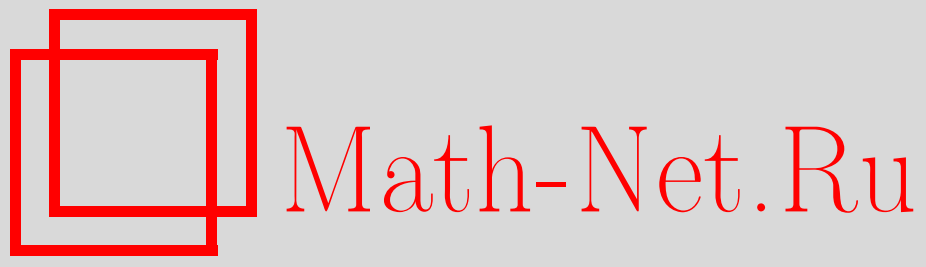

В. А. Курлин, Трехстраничные диаграммы Дынникова заузленных 3-валентных графов, Функи. анализ и его прил., 2001, том 35, выпуск 3, 84-88

DOI: https://doi.org/10.4213/faa264

Использование Общероссийского математического портала MathNet.Ru подразумевает, что вы прочитали и согласны с пользовательским соглашением

http://www . mathnet.ru/rus/agreement

Параметры загрузки:

IP : 54.157 .27 .8

26 апреля 2023 г., 04:12:24

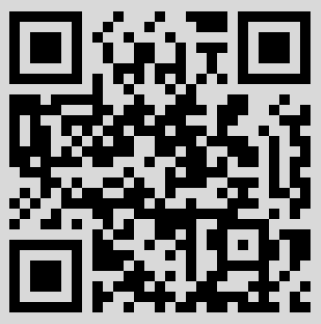


(1997). 7. Картан А., Эйленберг С. Гомологическая алгебра. М., ИЛ, 1960. 8. Ott C. Pacific J. Math., 173, №. 1, 173-179 (1996). 9. Туровский Ю. В. Спектральная теория операторов и ее прил., вып. 6, 144-181 (1985). 10. Frunza St. Banach Center Publ., 8, PWN, 281-287 (1982). 11. Wojtynski W. J. Func. Anal., 153, 405-413 (1998). 12. Бурбаки $H$. Спектральная теория. М., Мир (1972). 13. Досиев А. A. Функц. анализ и его прил., 34, вып. 4, 82-84 (2000). 14. Туровский Ю. В. Функц. анализ и его прил., 18, вып. 2, 77-78 (1984). 15. Beltita D. Stud. Math., 135, 163-178 (1999).

Институт математики и механики Академии наук Азербайджана Поступило в редакцию e-mail: info@sinam.net 26 октября 2000 г.

УДК 515.162.8

\title{
Трехстраничные диаграммы Дынникова заузленных 3-валентных графов
}

\author{
(c) 2001. В. А. Курлин
}

Недавно И. А. Дынников предложил новый способ кодирования неориентированных зацеплений с помощью трехстраничных диаграмм (см. [1]). С его помощью была получена алгебраическая классификация всех изотопических классов неориентированных зацеплений (см. [2]). Мы обобщаем этот подход на 3-валентные графы. Конечный граф называется 3-валентным, если из каждой его вершины выходят ровно три ребра. Мы рассматриваем только 3 -валентные неориентированные графы, в том числе несвязные, с петлями и мультиребрами. Под заузленным графом мы будем понимать вложение графа в $\mathbb{R}^{3}$, при котором ребра переходят в конечные ломаные. Будем исследовать заузленные графы с точностью до объемлемой изотопии. Объемлемая изотопия двух графов - это непрерывное семейство гомеоморфизмов $\phi_{t}: \mathbb{R}^{3} \rightarrow \mathbb{R}^{3}, t \in[0,1]$, такое, что $\phi_{0}=\mathrm{id}$, а $\phi_{1}$ переводит один из этих графов в другой. Изотопические инварианты заузленных графов изучались в $[3,4]$. Другие отношения эквивалентности, например конкордантность, гомотопность и гомологичность, см. в [5].

Понятие заузленного графа мотивировано с теоретической и практической точек зрения. Во-первых, задача изотопической классификации заузленных графов является частным случаем общей топологической проблемы классификации вложений в евклидово пространство. К тому же мы получаем естественное расширение классической теории узлов на более сложные одномерные объекты. Многие инварианты обычных зацеплений обобщаются на графы, например, полиномы Александера и инварианты Васильева (см. [6,7]). Как и обычные зацепления, заузленные графы легко изображать с помощью плоских диаграмм, которые определяются с точностью до следующих движений Райдемайстера:

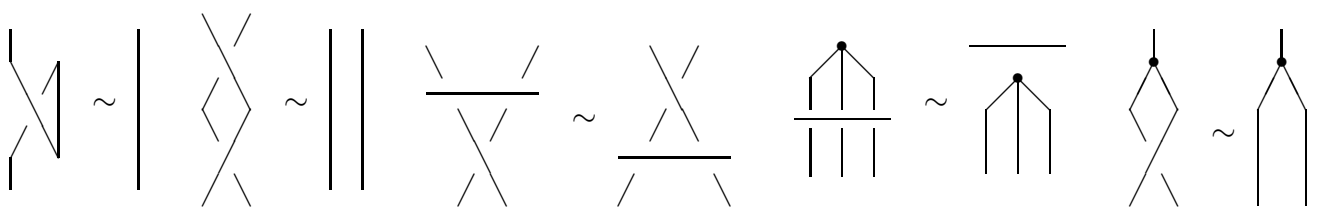


Во-вторых, заузленные графы являются математической моделью для длинных белковых соединений в органической химии и молекулярной биологии. Известно, что одной из устойчивых первичных структур молекулы ДНК, состоящей из нескольких сотен атомов, является скрученная $\alpha$-спираль (см. [8]). Устойчивость обусловлена действием водородных связей. Добавляя эти связи к молекуле в качестве виртуальных ребер, мы получим некоторый 3 -валентный граф в $\mathbb{R}^{3}$. Одна из главных проблем современной биологии - это предсказание реальной формы молекулы в зависимости от ее атомного состава (см. [9]). В частности, интересно, какие пространственные графы могут получиться для данной молекулы. Именно пространственная структура молекулы отвечает за ее разнообразные химические свойства. Например, большое значение имеет, может ли молекула принять форму, зеркально симметричную исходной [10]. Основная трудность - это абсолютная открытость любой биологической системы для действия внешних сил. Некоторые ферменты могут изменять топологический тип молекулы. Однако этим процессом можно управлять, что помогает при синтезе соединений определенного типа (см. [11]).

Далее наши рассуждения будут следовать работе [12]. Рассмотрим полугруппу $S t g$ с множеством образующих $\mathbb{A}=\left\{a_{i}, b_{i}, c_{i}, d_{i}, e_{i}, f_{i}, g_{i}, h_{i} ; i \in \mathbb{Z}_{3}\right\}$ и следующими 90 соотношениями $(i=1,2,3$; в силу соотношения $(3)$ одно из соотношений (4) можно выбросить):

(1) $a_{i}=a_{i+1} d_{i-1}, b_{i}=a_{i-1} c_{i+1}, c_{i}=b_{i-1} c_{i+1}, d_{i}=a_{i+1} c_{i-1}$,

(2) $e_{i}=d_{i} g_{i}=g_{i-1} b_{i+1}=a_{i+1} h_{i-1}, f_{i}=h_{i} b_{i}=d_{i+1} h_{i-1}=g_{i-1} c_{i+1}$,

(3) $d_{1} d_{2} d_{3}=1$

(4) $b_{i} d_{i}=d_{i} b_{i}=1$,

(5) $\left(d_{i-1} c_{i-1}\right) x=x\left(d_{i-1} c_{i-1}\right)$, где $x \in\left\{c_{i}, e_{i}, b_{i-1} d_{i} d_{i-1}\right\}$,

(6) $u v=v u$, где $u \in\left\{a_{i-1} b_{i-1}, e_{i-1} b_{i-1}, b_{i+1} d_{i-1} d_{i+1} b_{i-1}\right\}, v \in\left\{a_{i}, b_{i}, c_{i}, e_{i}\right.$, $\left.b_{i-1} d_{i} d_{i-1}\right\}$.

Теорема 1. Любой заузленный граф кодируется некоторым әлементом полугрупnь Stg.

ТеОрема 2. Заузленные графы изотопны тогда и только тогда, когда представляющие их әлементы совпадают в Stg.

Теорема 3. Элемент полугруппы Stg кодирует некоторый заузленный граф тогда и только тогда, когда он цеентральный, т. е. коммутирует с любым элементом этой полугруппы.

Теорема 3 доказывается аналогично лемме 3 из [2]. Пусть $P_{1}, P_{2}, P_{3}-$ три полуплоскости в $\mathbb{R}^{3}$, границы которых совпадают: $\partial P_{1}=\partial P_{2}=\partial P_{3}=\alpha$ (см. правую часть приведенного ниже рисунка). Положим $\mathbb{Y}=P_{1} \cup P_{2} \cup P_{3}$. На оси $\alpha$ выберем ориентацию. Трехстраничная диаграмма (3-диаграмма) заузленного графа $\Gamma$ - это такое вложение графа $\Gamma$ в $\mathbb{Y}$, что

(a) все основные вершины из $Г$ лежат на оси $\alpha$;

(b) (трансверсальность к $\alpha$ ) пересечение $\Gamma \cap \alpha=A_{1} \cup \cdots \cup A_{m}$ конечно;

(c) две дуги, примыкающие к вершине степени 2 , лежат в разных полуплоскостях;

(d) из трех дуг, примыкающих к 3-валентной вершине, две лежат в одной полуплоскости, третья лежит в другой;

(е) (монотонность) ограничение ортогональной проекции $\mathbb{R}^{3} \rightarrow \alpha \approx \mathbb{R}$ на каждую из связных компонент пересечения $\Gamma \cap P_{i}(a p \kappa y)$ является монотонной функцией для любого $i=1,2,3$. 
Доказательство теоремы 1. 3-диаграмма называется специальной, если каждая арка в полуплоскости $P_{2}$ имеет длину 2 , т. е. соединяет некоторую вершину $A_{i}$ с вершиной $A_{i+2}$, или длину 1 и тогда содержит 3 -валентную вершину. По плоской диаграмме графа построим специальную трехстраничную, определяющую тот же заузленный граф. Для каждого перекрестка плоской диаграммы $D$ отметим маленький отрезок верхней дуги (промежуточный мост), а для 3-валентной вершины - две выходящие из нее дуги (основной мост).

Выберем такой несамопересекающийся ориентируемый путь в плоскости диаграммы $D$ с концами вне $D$, чтобы он проходил по каждому мосту ровно один раз и пересекал оставшуюся часть диаграммы трансверсально. Деформируем плоскость диаграммы так, чтобы этот путь стал отрезком некоторой прямой $\alpha$. Затем приклеим вдоль этой прямой полуплоскость $P_{2}$. Вытолкнем в $P_{2}$ каждый промежуточный мост в виде одной тривиальной арки, а каждый основной - в виде двух арок с 3-валентной вершиной на стыке:

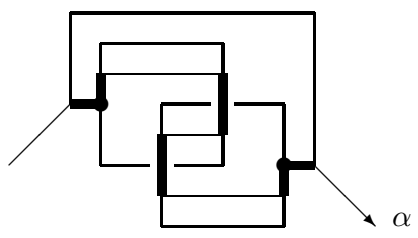

$D$

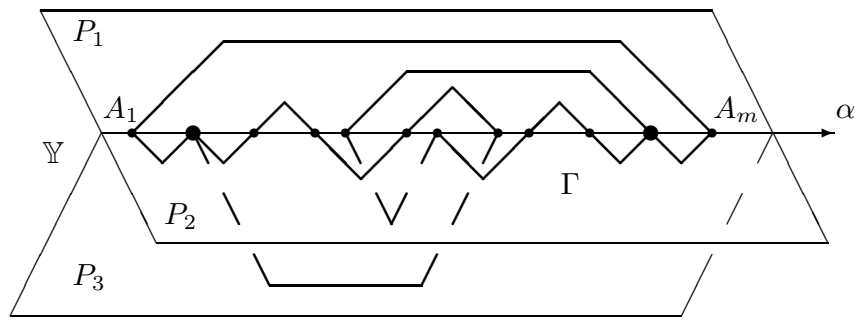

Очевидно, что всю 3-диаграмму заузленного графа можно однозначно восстановить по части диаграммы в малой окрестности оси $\alpha$. Для этого достаточно в каждой полуплоскости соединять звенья, направленные друг к другу, начиная с самых внутренних. 3-диаграмма в окрестности $\alpha$ может состоять из элементов 24 типов:

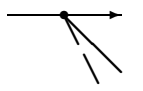

$a_{1}$

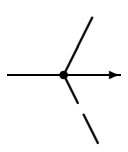

$a_{2}$

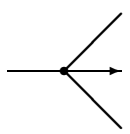

$a_{3}$

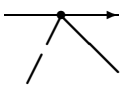

$b_{1}$

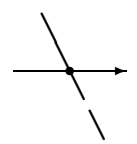

$b_{2}$

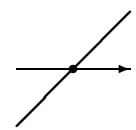

$b_{3}$

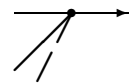

$c_{1}$

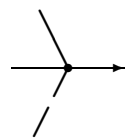

$c_{2}$

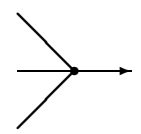

$c_{3}$

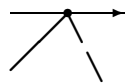

$d_{1}$

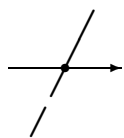

$d_{2}$

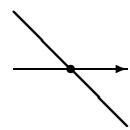

$d_{3}$

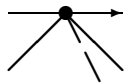

$e_{1}$

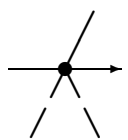

$e_{2}$

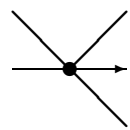

$e_{3}$

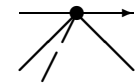

$f_{1}$

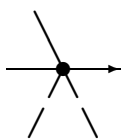

$f_{2}$

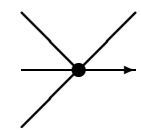

$f_{3}$

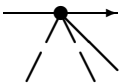

$g_{1}$

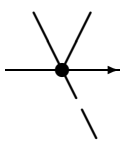

$g_{2}$

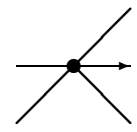

$g_{3}$

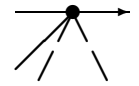

$h_{1}$

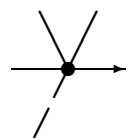

$h_{2}$

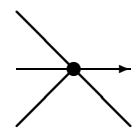

$h_{3}$

Пусть $W-$ множество всех слов в таком алфавите $\mathbb{A}$, включая пустое слово $\varnothing$. Для данной 3-диаграммы $Г$ выпишем последовательно типы всех вершин на оси $\alpha$. Получим слово $w_{\Gamma} \in W$ (для изображенной выше диаграммы $\left.w_{\Gamma}=a_{3} e_{1} b_{3} d_{3} a_{2} b_{3} b_{1} c_{2} b_{3} d_{3} h_{3} c_{3}\right)$. Заметим, что таким образом получаются не все 
слова из $W$, а только сбалансированные. Есть простой геометрический критерий сбалансированности слова: все звенья, выходящие из точек на оси, должны состыковываться друг с другом. Это легко записать и алгебраически через алфавит $\mathbb{A}$. Для несбалансированных слов звенья соответствующей 3-диаграммы могут выходить на ее край, не соединяясь с другими.

Набросок доказательства теоремы 2. Все соотношения (1)-(6) легко реализуются изотопией в $\mathbb{R}^{3}$. Эту изотопию можно провести в двумерном комплексе $\mathbb{T}$, полученном из $\mathbb{Y}$ приклейкой полуплоскости $P_{4}$ к $P_{1} \cup P_{2}$ по прямой, перпендикулярной $\alpha$. Любая 3 -диаграмма редуцируется к специальной, согласно следующему утверждению, которое доказывается аналогично предложению 2 в [1].

ЛЕмма 1. Любое сбалансированное слово для любого $i=1,2,3$ разлагается с помощью соотношений (1)-(6) на следующие подслова: $a_{i}, b_{i}, c_{i}, d_{i}, e_{i}$, $b_{i-1} b_{i} d_{i-1}, b_{i-1} d_{i} d_{i-1}$.

Осталось показать, что соотношений (1)-(6) достаточно для реализации изотопии графов. Введем полугруппу Sit бесконечных плетений. Расположим две положительные оси $\mathbb{R}_{+}$в $\mathbb{R}^{3}$ горизонтально друг над другом и обозначим через $V$ трехмерный слой между горизонтальными плоскостями, в которых лежат эти оси. Рассмотрим 3-валентный граф $T$, содержащий конечное число 3 -валентных вершин и счетное число изолированных отрезков. Назовем бесконечным плетением вложение графа $T$ в указанный слой $V$, такое, что концевые вершины графа $T$ совпадают с натуральными точками на осях $\mathbb{R}_{+}$. Мы предполагаем, что достаточно далеко от нулевого края все отрезки плетения параллельны друг другу, но могут не быть вертикальными. Отношение эквивалентности на таких графах объемлемая изотопия в $V$, неподвижная на границе слоя. Произведение плетений $T_{1} T_{2}$ получается приклейкой верхней оси $\mathbb{R}_{+}$плетения $T_{2}$ к нижней оси $\mathbb{R}_{+}$плетения $T_{1}$. Так мы получаем структуру полугруппы Sit с единицей 1 , состоящей из вертикальных отрезков. Следующая лемма доказывается аналогично теореме Райдемайстера. Фактически соотношения (7)-(15) геометрически отвечают переходу через особенности коразмерности 1 в пространстве всех бесконечных плетений с самопересечениями.

Лемма 2. Полугруппа Sit порождена образующими $u_{k}, v_{k}, \sigma_{k}^{ \pm 1}, \lambda_{k}, y_{k}$,
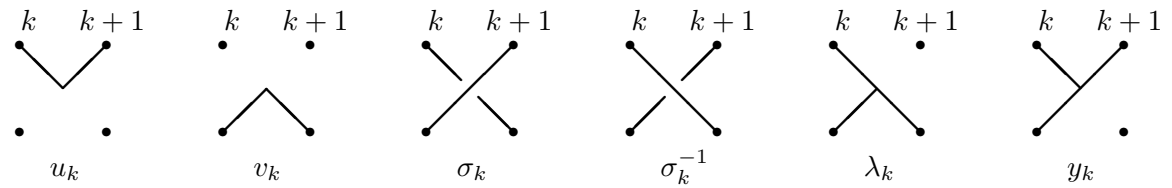

и соотношениями $(k+1<l)$

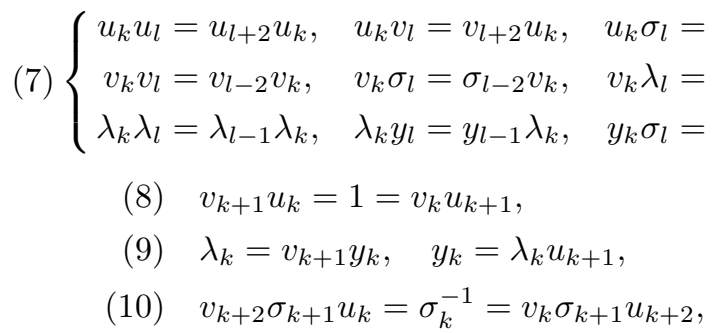
$\begin{array}{rlrl}u_{k} \lambda_{l} & =\lambda_{l+2} u_{k}, & u_{k} y_{l} & =y_{l+2} u_{k}, \\ v_{k} y_{l}=y_{l-2} v_{k}, & \lambda_{k} \sigma_{l}=\sigma_{l-1} \lambda_{k}, \\ y_{k} y_{l}=y_{l+1} y_{k}, & \sigma_{k} \sigma_{l}=\sigma_{l} \sigma_{k},\end{array}$

$$
\begin{aligned}
& v_{k} \sigma_{k}=v_{k}, \quad \sigma_{k} u_{k}=u_{k} \\
& \sigma_{k} \sigma_{k}^{-1}=1=\sigma_{k}^{-1} \sigma_{k} \\
& \sigma_{k} \sigma_{k+1} \sigma_{k}=\sigma_{k+1} \sigma_{k} \sigma_{k+1}
\end{aligned}
$$




$$
\begin{aligned}
& \lambda_{k} \sigma_{k+1} \sigma_{k}=\sigma_{k} \lambda_{k+1}, \sigma_{k} \sigma_{k+1} y_{k}=y_{k+1} \sigma_{k}, \sigma_{k} \lambda_{k}=\lambda_{k+1} \sigma_{k} \sigma_{k+1}, y_{k} \sigma_{k}=\sigma_{k+1} \sigma_{k} y_{k+1}, \\
& \lambda_{k} \sigma_{k}=\lambda_{k}, \quad \sigma_{k} y_{k}=y_{k} . \quad \square
\end{aligned}
$$

Любое бесконечное плетение можно преобразовать в 3-диаграмму, поставив его оси $\mathbb{R}_{+}$вертикально, приклеив к полученному нижнему краю $\mathbb{R} \times 0$ две полуплоскости и стянув все перекрестки и 3 -валентные вершины на ось $\mathbb{R} \times 0$. Это задает вложение полугруппы бесконечных плетений Sit в полугруппу Stg. В частности, образующим соответствуют элементарные слова: $u_{k}=d_{3}^{k} c_{3} b_{3}^{k-1}$, $v_{k}=d_{3}^{k-1} a_{3} b_{3}^{k}, \sigma_{k}=d_{3}^{k-1} b_{2} d_{3} d_{2} b_{3}^{k}, \lambda_{k}=d_{3}^{k-1} e_{3} b_{3}^{k}, y_{k}=d_{3}^{k} f_{3} b_{3}^{k-1}$. Любое сбалансированное слово разлагается на элементарные аналогично тому, как это делается в [12], т. е. такое слово определяет некоторое бесконечное плетение. Поскольку каждый заузленный граф представляется сбалансированным словом, ему можно поставить в соответствие бесконечное плетение. При этом изотопным заузленным графам отвечают изотопные бесконечные плетения. Чтобы закончить доказательство теоремы 2, осталось вывести соотношения (7)-(15) полугруппы Sit из соотношений (1)-(6) полугруппы Stg. Разложение на элементарные слова и вывод соотношений аналогичны соответствующим разложениям в [12].

Автор выражает благодарность своему научному руководителю профессору В. М. Бухштаберу за поддержку и доценту И. А. Дынникову за внимание к работе.

\title{
ЛИТЕРАТУРА
}

1. Дынников И. А. Функц. анализ и его прил., 33, вып. 4, 25-37 (1999). 2. Дынников И. А. Функц. анализ и его прил., 34, вып. 1, 29-40 (2000). 3. Kauffman L. Trans. Amer. Math. Soc., 311, No. 2, 697-710 (1989). 4. Jonish, D., Millett, K. C. Trans. Amer. Math. Soc., 327, No. 2, 655-702 (1991). 5. Taniyma K. Topology, 33, No. 3, 509-523 (1994). 6. Litherland R. Math. Proc. Cambridge Philos. Soc., 106, No. 1, 95-106 (1989). 7. Stenford T. Topology, 35, No. 4, 1027-1050 (1996). 8. Франк-Каменецкий Самая главная молекула (Библиотечка «Квант»). 9. Альбертс и др. Молекулярная биология клетки, т. 1. 10. Шилл Г. Катенаны, ротоксаны и узлы. Мир, М., 1973. 11. Simon J. Topology, 25, 229-235 (1986). 12. Дынников И. А. Конечно-определенные полугруппы и группы в теории узлов. Труды МИАН, т. 231.

Московский государственный университет

Поступило в редакцию 22 июня 2000 г.

УДК 517.535.2

\section{Модули эллиптических кривых и числа вращения диффеоморфизмов окружности}

\author{
(c) 2001. В. С. МолдАвСКий
}

Комплексное возмущение аналитического диффеоморфизма окружности - это биголоморфное отображение, фундаментальная область которого - эллиптическая кривая. В. И. Арнольд предположил, что модуль этой кривой стремится к числу вращения исходного диффеоморфизма при стремлении к нулю параметра 